\title{
EFEITOS DO BENEFICIAMENTO NA QUALIDADE DE SEMENTES DE MILHO INFECTADAS POR Fusarium moniliforme Sheld
}

\author{
M.L.M. de CARVALHO; D.A.C. BILIA; W.R. da SILVA \\ Deparlamento de Agricultura - ESALQ/USP - C.P. 9 - CEP: 13418-900 - Piracicaba,SP \\ J.R. de MENEZES \\ Departamento de Futopatologia - FUEL - CEP: 86001-000 - Londrina,PR.
}

RESUMO: Com o objetivo de avaliar os efeitos do beneficiamento na qualidade de lotes com sementes de millo infectadas com Fusarium monilforme Sheld, sementes foram submetidas à classificação por peneiras, originando 4 frações $(24 / 64 ; 22 / 64 ; 20 / 64 ; 18 / 64$ de polegada) e à separação por peso através da ação da mesa gravitacional, originando três frações que foram individualmente submetidas ao mesmo equipamento gerando, cada uma, mais três fraçōes. Todas as fraçōes foram avaliadas quanto à germinação, ao vigor, à densidade, ao peso de mil sementes e à sanidade. A classificação por largura não é eficaz para separar as sementes infectadas; no entanto, o aumento da largura das sementes é acompanhado pela elevaçāo dos valores da germinação e do vigor.

Descritores: milho, sementes, beneficiamento, $F$. moniliforme.

\section{PROCESSING EFFECTS ON THE QUALITY OF CORN SEED INFECTED BY Fusarium moniliforme SAladD}

\begin{abstract}
In order to evaluate processing effects on the quality of corn seeds infected by Fusarium moniliforme Sheld seeds were submitted to screen classification yielding 4 fractions $(24 / 64 ; 22 / 64 ; 20 / 64 ; 18 / 64$ of the inch) and the other part was separated by weight in a gravity separator. The separator originated three fractions that in turn were individually submitted to the same equipment generating, each one, three other fractions. All fractions were evaluated for germination, vigor, density, weight of a thousand seeds, and sanity. The width classification is not effective in the selection of infected seeds; however, the increase of seed width is followed by higher germination and vigor values.
\end{abstract}

Key Words: corn, seeds, improvement, F.moniliforme.

\section{INTRODUÇÃO}

Diversas doenças, muitas das quais transmitidas pelas sementes, têm sido apontadas como redutoras da produtividade na cultura do milho. LUCCA FILHO (1984a) afirma que, dentre os fungos frequentemente encontrados em sementes de milho, os mais importantes em termos de qualidade para semeadura são Fusarium moniliforme, Diplodia maydis, Penicilliun sp. e Aspergillus spp., uma vez que podem contribuir para a redução do poder germinativo.

A ocorrência de Fusarium moniliforme em milho, está associada a fatores diversos. DJAKAMIHARDJA et al. (1970) citam que, nos EUA, este é o fungo que mais ocorre em áreas úmidas.
Da mesma forma, SALAMA \& MISHRICKY (1973) trabalhando com diferentes cultivares de milho obtidas no Egito, afirmaram que Fusarium moniliforme foi o fungo predominante em todos os materiais, embora houvesse variação de ocorrência entre os mesmos.

No Brasil, não são relatados casos de ataques severos de Fusarium moniliforme em lavouras de milho.

No entanto, na safra 1990/91 foi detectada, na região norte do Mato Grosso, incidência acentuada desse fungo em campos de produção de sementes.

O efeito da presença do fungo na semente é controvertido, principalmente no que se refere à sua influência na germinação. Autores como MELCHERS \& JOHNSTON (1924), 
WARMKE \& SCHENCK (1971) e BEDENDO (1978), não encontraram uma correlação entre a presença de F.moniliforme e a redução da germinação das sementes.

Por outro lado, CUDDY \& WALLEN (1965) constataram que lotes com $62 \%$ de sementes infectadas com Fusarium moniliforme apresentaram germinação de $28 \%$, enquanto lotes de sementes sadias mostraram $94 \%$ de germi ação.

FUTREL \& KILGORE (1969) observaram que os danos causados por esse fungo podem reduzir o desenvolvimento de plântulas e afetar o "stand" inicial da cultura.

MENEZES (1988) afirma que o uso de sementes infectadas ou infestadas com Fusarium poderá afetar negativamente a germinação, a emergência e a produção, além de colaborar efetivamente para a disseminação do organismo no campo; ressalta, ainda, que nem todas as espécies de Fusarium presentes nas sementes são patogênicas, citando que $F$. moniliforme pode estar presente na semente, sem prejudicar, aparentemente, a germinação.

Segundo FUTREL \& KILGORE (1969) e SCOTT \& FUTREL (1970) o fungo atua, sobre a germinação e o desenvolvimento das plântulas, sintetizando uma toxina no endosperma da semente de milho que, translocada para as raízes, provoca a inibição do seu desenvolvimento.

A remoção de materiais indesejáveis de um lote de sementes pode ser conseguida, através do beneficiamento, pelas diferenças físicas entre os componentes. LOLLATO \& SILVA, (1984) concluiram que a utilização da mesa gravitacional permitiu separar sementes de feijão em função das suas características relacionadas com densidade, peso unitário, germinação, vigor, sanidade, pureza física, manchas e defeitos.

Considerando que $o$ ataque de microrganismos pode afetar o peso, o tamanho e a qualidade das sementes, o objetivo do presente trabalho foi o de averiguar o efeito do beneficiamento na qualidade das sementes de milho portadoras de Fusarium moniliforme.

\section{MATERIAL E MÉTODOS}

O presente ensaio foi conduzido no Laboratório de Análise de Sementes e de Patologia de Sementes da Escola Superior de Agricultura "Luiz de Queiroz", no período de abril a junho de 1991.

Sementes de milho, produzidas na região norte do Mato Grosso, com incidência de manchas e oriundas de espigas deformadas, foram submetidas a exame patológico prévio para determinação do agente causal da doença através do método de papel de filtro (ISTA, 1976).

Os danos causados na espiga foram avaliados de acordo com a estimativa percentual da área afetada com sintomas visiveis, utilizando a classificação apresentada na TABELA 1.

TABELA 1 - Classificação empregada na estimativa da área da espiga afetada com danos visíveis de origem patogênica.

\begin{tabular}{cc}
\hline Classe de dano & Área afetada (\%) \\
\hline 1 & Inferior a 1 \\
2 & 1 a 10 \\
3 & 11 a 20 \\
4 & 21 a 40 \\
5 & 41 a 60 \\
6 & 61 a 80 \\
7 & Superior a 80 \\
\hline
\end{tabular}

Após a realização da pré-limpeza com a utilização das peneiras 11/64" (desfolha) e 28/64" (peneiração) foi obtido o lote denominado "original" $(O)$.

A seguir, as sementes foram separadas por largura e por peso em opcrações independentes.

A separação por largura foi cfetuada por peneiras dispostas em série decrescente $(24 / 64$, $22 / 64,20 / 64$ e 18/64 de polegada), dando origem às quatro frações correspondentes.

A separação por peso, realizada $\mathrm{cm}$ mesa gravitacional, originou 12 frações (F) conforme o indicado na Figura 1.

$O$ lote original $(O)$ proveniente da prélimpeza, e as frações originadas das separações por largura e peso, foram avaliados quanto às qualidades físicas, fisiologicas e sanitárias, através das seguintes determinações:

a) Peso de mil sementes: conforme recomendações de BRASIL (1980) pelo método de pesagem direta das amostras com mil sementes.

b) Densidade das sementes: foi obtida com o emprego do volumenômetro, descrito por KIEHL 
(1979), utilizando 5 sementes e $15 \mathrm{ml}$ de areia por repetição.

c) Teste de germinação: realizado de acordo com as Regras para Análise de Sementes (BRASIL, 1980 ), empregando temperatura de $30^{\circ} \mathrm{C}$.

d) Teste de vigor: a emergência em areia e o envelhecimento acelerado foram executados conforme metodologia descrita por POPINIGS (1977). O teste de frio sem solo foi efetuado conforme FRATIN (1987).

e) Sanidade: foi utilizado o método do papel de filtro com congelamento e desinfecção superficial das sementes com hipoclorito de sódio $1 \%$, por 5 minutos, de acordo com as recomendações da ISTA (1976).

A análise estatística foi efetuada adotando delineamento experimental inteiramente casualizado, com quatro repetições, e o auxílio do programa Sistema de Análise Estatística (ZONTA et al., 1984). A comparação de médias foi feita pelo teste de Tukey, ao nível de $5 \%$ de probabilidade.

\section{LOTE ORHGINAL (0)}

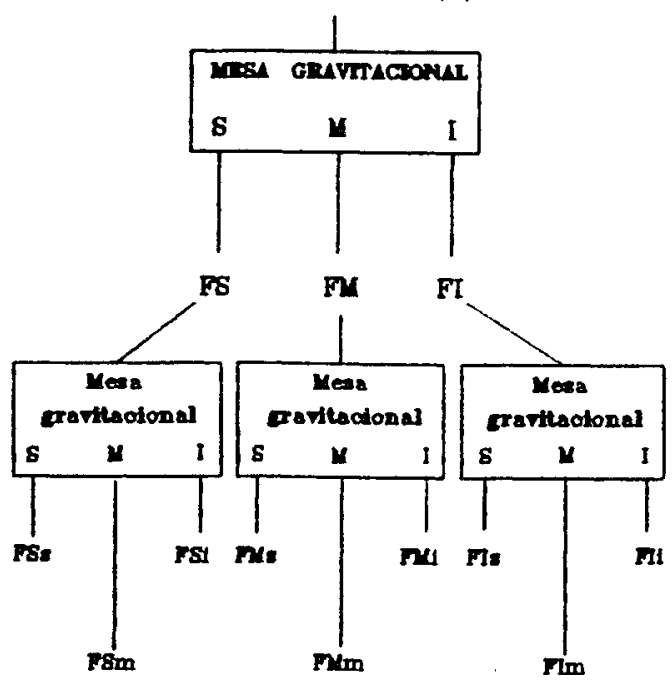

Figura 1 - Esquema da separação por peso realizada em mesa gravitacional. Piracicaba, 1991.

\section{RESULTADOS E DISCUSSÃO}

O patógeno presente nas sementes foi identificado como sendo Fusarium moniliforme Sheld. De acordo com LUCCA FILHO (1987a) as sementes de milho portadoras de Fusarium moniliforme Sheld apresentam estrias no endosperma, como sendo o sintoma mais comum.

O mesmo autor afirma, ainda, que o referido patógeno provoca podridão nas sementes e nas espigas, promovendo o aparecimento, entre as sementes, de um micélio cotonoso, com esporos, conferindo uma coloração que varia de rosa a marrom-avermelhada. Além dos sintomas reportados por LUCCA FILHO (1987b), foram observadas na avaliação sintomatológica, manchas circulares de bordos escuros e centro claro.

A. TABELA 2, mostrando os dados obtidos na avaliação de espigas com sintomas de Fusarium moniliforme, indica que as classes $2,3 \mathrm{e}$ 4 (1 a $40 \%$ de área afetada) abrigaram a maioria $(83,95 \%)$ dos casos avaliados. Este fato confirma a ampla disseminação do patógeno na população estudada.

TABELA 2 - Frequência dos danos causados às espigas de milho por Fusarium moniliforme Sheld. Piracicaba, 1991.

\begin{tabular}{ccc}
\hline \hline Classe de dano & $N^{0}$ de espigas & \% de espigas \\
\hline 1 & 50 & 4,43 \\
2 & 573 & 50,73 \\
3 & 248 & 21,79 \\
4 & 129 & 11,43 \\
5 & 74 & 6,55 \\
6 & 36 & 3,19 \\
7 & 19 & 1,68 \\
\hline Total & 1.129 & 100,00 \\
\hline
\end{tabular}

Na TABELA 3, estão os resultados obtidos nas determinações do pelo de mil sementes, da densidade, da germinação, do vigor e da sanidade das sementes classificadas pela largura. A observação dos dados do peso de mil sementes permite verificar que a separação por largura mostrou diferença significativa entre as frações, indicando uma tendência de redução do peso de sementes com a redução do tamanho. Quanto à densidade, foi observada uma tendência inversa; neste caso, as sementes retidas em peneiras inferiores apresentaram valores superiores aos das demais. 
TABELA 3 - Dados médios das determinações relativas às qualidades físicas, fisiológicas e sanitárias em sementes classificadas pela largura. Piracicaba, 1991.

\begin{tabular}{lcccccc}
\hline \hline & & \multicolumn{5}{c}{ Tratamentos } \\
\cline { 3 - 7 } \multicolumn{1}{c}{ Determinações } & CV\% & Original & 24 & 22 & 20 & 18 \\
\hline Peso 1000 $(\mathrm{g})$ & 2,5 & $288,400 \mathrm{c}$ & $347,225 \mathrm{a}$ & $304,175 \mathrm{~b}$ & $263,700 \mathrm{~d}$ & $200,500 \mathrm{c}$ \\
Densidade (g/cm $)$ & 12,0 & $1,161 \mathrm{~b}$ & $1,157 \mathrm{~b}$ & $1,173 \mathrm{~b}$ & $1,208 \mathrm{ab}$ & $1,521 \mathrm{a}$ \\
Germinação (\%) & 1,8 & $75 \mathrm{~b}$ & $85 \mathrm{a}$ & $75 \mathrm{~b}$ & $68 \mathrm{c}$ & $52 \mathrm{~d}$ \\
Emergência (\%) & 4,9 & $80 \mathrm{a}$ & $84 \mathrm{a}$ & $79 \mathrm{a}$ & $68 \mathrm{~b}$ & $56 \mathrm{c}$ \\
Env. Acel. (\%) & 3,7 & $50 \mathrm{~b}$ & $70 \mathrm{a}$ & $66 \mathrm{a}$ & $56 \mathrm{~b}$ & $41 \mathrm{c}$ \\
Frio s/solo (\%) & 2,6 & $74 \mathrm{~b}$ & $81 \mathrm{a}$ & $78 \mathrm{a}$ & $60 \mathrm{c}$ & $53 \mathrm{~d}$ \\
Infecção (\%) & 6,4 & $89 \mathrm{a}$ & $90 \mathrm{a}$ & $89 \mathrm{a}$ & $88 \mathrm{a}$ & $93 \mathrm{a}$ \\
\hline \hline
\end{tabular}

Em cada linha, médias seguidas por letras iguais, não diferem entre si pelo teste de Tukey, ao nível de $5 \%$ de probabilidade.

SILVA \& MARCOS FILHO (1979), trabalhando com sementes de milho, obtiveram, através da separação por tamanho, materiais semelhantes quanto à densidade.

Os resultados do teste de germinação indicam que os tratamentos, que incluiram sementes de maior tamanho, apresentaram valores absolutos superiores, apesar de não se diferenciarem dos demais tratamentos quanto à incidência de microrganismos. A superioridade na germinação das sementes retidas na peneira $24 \mathrm{em}$ relação à das demais, também foi encontrada por SCOTTI \& KRZYZANOWSKI (1977); por outro lado SILVA \& MARCOS FILHO (1979) obtiveram resultados semelhantes apenas em uma das cultivares estudadas. Nos testes de vigor (emergência em areia, envelhecimento acelerado e frio sem solo), a separação por tamanho resultou na superioridade para as sementes retidas nas peneiras 24 e 22 em relação às demais.

Dados semelhantes foram encontrados por BARNES (1959), quando trabalhou com o teste de frio em sementes de milho. No entanto, COSTA \& CARVALHO (1983), empregando o envelhecimento acelerado em sementes de milho, não observaram uma influência significativa do tamanho da semente no seu vigor. O teste de sanidade mostrou alta incidência de Fusarium moniliforme nas sementes de todos os tamanhos; a análise estatística não detectou diferenças significativas entre os tratamentos, o que demonstra que a classificação por tamanho não foi capaz de separar sementes infectadas por $F$. moniliforme. Todavia, não foram efetuadas observações sobre a severidade da doença nos indivíduos.

$\mathrm{Na}$ tentativa de separação por peso com o emprego da mesa gravitacional, foi considerada a especificidade de ação de equipamento para esta finalidade. Dessa maneira, a separação realizada no lote original $(O)$ levou à obtenção de três frações que, individualmente reconduzidas à mesa gravitacional, vieiram a atuar como novos lotes originais (FS, FM, FI) nas separações posteriores.

Por esse motivo, as comparações efetuadas adotam os lotes originais, relativos à cada uma das separações, como referências para a interpretação dos resultados (TABELAS 4,5 e 6).

A TABELA 4, referente à qualidade física, permite verificar que a mesa gravitacional não foi eficiente na separação por peso. A determinação da densidade dentro de lotes, não mostrou diferenças significativas. Dentro das frações, apenas a fração média apresentou diferenças significativas, sendo que as sementes da descarga superior apresentaram maior densidade quando comparadas às demais.

Dados obtidos por SILVA (1978) indicaram que a mesa gravitacional foi eficiente na 
separação pelos pesos unitários e volumétricos de sementes de milho.

TABELA 4 - Dados médios do peso de mil sementes $(P .1000)$ e de densidade das sementes submetidas à passagem em mesa gravitacional. Piracicaba, 1991.

\begin{tabular}{ccc}
\hline & \multicolumn{2}{c}{ Determinaçōes } \\
\cline { 2 - 3 } Tratamento & P 1000 $(\mathrm{g})$ & Densidade $\left(\mathrm{g} / \mathrm{c}^{3}\right)$ \\
\hline O & $284,075 \mathrm{a}$ & $1,161 \mathrm{a}$ \\
FS & $284,700 \mathrm{a}$ & $1,270 \mathrm{a}$ \\
\hline FM & $288,450 \mathrm{a}$ & $1,410 \mathrm{a}$ \\
\hline FI & $282,250 \mathrm{a}$ & $1,243 \mathrm{a}$ \\
\hline CV\% & 2,2 & 13,9 \\
\hline FS & $284,700 \mathrm{~b}$ & $1,270 \mathrm{a}$ \\
\hline FSs & $302,025 \mathrm{a}$ & $1,211 \mathrm{a}$ \\
\hline FSm & $286,575 \mathrm{~b}$ & $1,275 \mathrm{a}$ \\
\hline FSi & $288,899 \mathrm{~b}$ & $1,237 \mathrm{a}$ \\
\hline CV\% & 1,6 & 13,7 \\
\hline FM & $288,450 \mathrm{ab}$ & $1,310 \mathrm{ab}$ \\
\hline FMs & $286,149 \mathrm{ab}$ & $1,492 \mathrm{a}$ \\
\hline FMm & $294,825 \mathrm{a}$ & $1,111 \mathrm{c}$ \\
\hline FMi & $281,950 \mathrm{~b}$ & $1,198 \mathrm{bc}$ \\
\hline FV\% & 2,0 & 9,1 \\
\hline FIs & $283,075 \mathrm{a}$ & $1,243 \mathrm{a}$ \\
\hline
\end{tabular}

Em cada coluna, médias seguidas por letras iguais não diferem entre si, pelo teste de Tukey ao nível de $5 \%$ de probabilidade.

A diferença de resultados no presente ensaio, pode ser atribuída às variações de metodo- logia, uma vez que o pequeno volume de material disponível exigiu a utilização de uma mesa gravitacional de pequeno tamanho; da mesma forma, a heterogeneidade de tamanho entre as sementes dos lotes originais pode ter contribuído para dificultar a ação da mesa gravitacional.

Pela TABELA 5 pode ser observado que, nos lotes resultantes da primeira separação por peso, a porcentagem de germinação da porção média foi superior à das porções inferiores e superiores. Já, para as frações, a germinação das sementes das descargas superiores foi, invariavelmente, a mais elevada. Os dados dos testes de vigor não permitiram, em razão de sua variabilidade, interpretação que pudesse sugerir a existência de quaisquer tendências.

O teste de sanidade para as sementes submetidas à mesa gravitacional (TABELA 6), não mostrou diferenças significativas entre os tratamentos, indicando que a classificação efetuada pelo equipamento não foi efetiva na separação das sementes infectadas. $O$ mesmo não ocorreu com sementes de feijão contaminadas por Fusarium sp. (LOLLATO \& SILVA, 1984); neste caso, as sementes sadias tenderam a se concentrar nas descargas superiores da mesa gravitacional.

A separação através de peneiras, apesar de não ter sido eficiente em separar sementes infectadas por Fusarium moniliforme mostrou que as sementes de maior largura apresentaram maiores valores para os testes de germinação e vigor. $A$ ineficiência na separação pode estar ligada ao método utilizado na detecção do patógeno que, apesar de identificar a presença de Fusarium moniliforme nas sementes de milho, não avalia a severidade de infecção nas unidades.

A separação por peso, pretendida com o uso da mesa gravitacional não foi efetiva, o que dificultou qualquer conclusão relativa ao peso das sementes.

\section{CONCLUSÕES}

1) A classificação de sementes de milho por largura, não é eficaz para separar, do lote, as unidades infectadas por Fusarium moniliforme Sheld.

2) O aumento da largura da semente é acompanhado da elevação dos valores de germinação e vigor. 
TABELA 5 - Dados médios obtidos nos testes de germinação (\%G), envelhecimento acelerado (E.A. \%), emergência (\%E) e de frio (\%F) em sementes submetidas à passagem em mesa gravitacional. Piracicaba, 1991.

\begin{tabular}{|c|c|c|c|c|}
\hline \multirow[t]{2}{*}{ Tratamentos } & \multicolumn{4}{|c|}{ Testes } \\
\hline & $\% G$ & EA\% & $\% \mathrm{E}$ & $\% \mathrm{~F}$ \\
\hline 0 & $81 a$ & $55 \mathrm{~b}$ & $78 a$ & $68 b$ \\
\hline FS & $73 b$ & $59 \mathrm{ab}$ & $80 a$ & $70 \mathrm{ab}$ \\
\hline FM & $82 a$ & $62 a$ & $77 a$ & $74 b$ \\
\hline FI & $74 b$ & $61 a b$ & $78 a$ & $72 \mathrm{ab}$ \\
\hline CV\% & 2,5 & 5,2 & 4,8 & 2,9 \\
\hline FS & $73 b$ & $58 b c$ & $80 \mathrm{bc}$ & $70 c$ \\
\hline FSs & $80 a$ & $57 \mathrm{c}$ & $87 a$ & $76 a b$ \\
\hline FSm & $81 a$ & $61 \mathrm{a}$ & $79 c$ & $74 b$ \\
\hline FSs & $75 b$ & $64 a$ & $85 a b$ & $79 a$ \\
\hline CV\% & 13,4 & 1,4 & 3,2 & 1,7 \\
\hline FM & $82 a$ & $62 a$ & $77 a$ & $74 b$ \\
\hline FMs & $78 b$ & $65 a$ & $80 a$ & $77 a$ \\
\hline FMm & $76 \mathrm{~b}$ & $56 \mathrm{~b}$ & $76 a$ & $73 b$ \\
\hline FMi & $68 c$ & $48 c$ & $71 a$ & $69 c$ \\
\hline CV\% & 8,9 & 4,4 & 7,6 & 1,5 \\
\hline FI & $74 b$ & $61 a$ & $78 a$ & $72 b$ \\
\hline FIs & $75 \mathrm{ab}$ & $62 a$ & $79 a$ & $76 a$ \\
\hline FIm & $77 a$ & $56 b$ & $80 a$ & $74 a$ \\
\hline Fli & $69 c$ & $57 b$ & $73 a$ & $64 c$ \\
\hline CV\% & 8,2 & 3,2 & 6,2 & 1,5 \\
\hline
\end{tabular}

Em cada coluna, médias seguidas por letras iguais não diferem entre si, pelo teste de Tukey, ao nível de $5 \%$ de probabilidade. 
Tabela 6 - Teste de sanidade para detecção de Fusarium moniliforme em lotes e frações classificadas em mesa gravitacional. Piracicaba, 1991.

\begin{tabular}{|c|c|}
\hline Tratamento & $\%$ de Infecção \\
\hline $\mathrm{O}$ & $89 \mathrm{a}$ \\
\hline FS & $91 \mathrm{a}$ \\
\hline FM & $94 \mathrm{a}$ \\
\hline FI & $94 a$ \\
\hline $\mathrm{CV} \%$ & 91 a \\
\hline FS & 91 a \\
\hline FSs & 94 a \\
\hline FSm & 93 a \\
\hline FSi & 95 a \\
\hline CV\% & 5,0 \\
\hline FM & 94 a \\
\hline FMs & $93 a$ \\
\hline FMm & 92 a \\
\hline FMi & $91 \mathrm{a}$ \\
\hline CV\% & 2,2 \\
\hline FI & 94 a \\
\hline FIs & $91 \mathrm{a}$ \\
\hline FIm & 94 a \\
\hline Fli & $94 a$ \\
\hline CV\% & 5,3 \\
\hline
\end{tabular}

Em cada coluna, médias seguidas por letras iguais não diferem entre si, pelo teste de Tukey, ao nível de $5 \%$ de probabilidade.

\section{REFERÊNCIAS BIBLIOGRÁFICAS}

BARNES, R.F. Seed size has influence on sweet corn maturity. Crops and Soils, Madison, v.12, n.3, p.2122, 1959.

BEDENDO, 1.P. Metodologia para a detecção de Fusarium moniliforme Sheld. e sua ocorrência em sementes de milho (Zea mays L.) produzidas no Estado de São Paulo. Piracicaba, 1978. Dissertação (Mestrado) - Escola Superior de Agricultura "Luiz de Queiroz", Universidade de São Paulo.
BRASIL, Ministério da Agricultura. Regras para análise de sementes. Brasília: Departamento Nacional de Produção Vegetal, 1980. 182p.

COSTA, C.L.V.; CARVALHO, N.M. Efeito do tamanho sobre o comportamento de sementes de milho submetidas ao envelhecimento artificial. Revista Brasileira de Sementes, Brasilia, v.5, p.23-29, 1983.

CUDDY, T.F.; WALLEN, V.R. Seed-borne disease of corn in 1964 and their effect on germination. Canadian Plant Disease Survey, Ottawa, v.45, p.3334, 1965

DJAKAMIHARDJA, G.; SCOTT, G.T.; FUTRELL, M.C. Seedling reaction of inbreds and single crosses of maize to Fusarium moniliforme. Plant Disease Reporter, Beltsville, v.54, p.307-310, 1970.

FRATIN, P. Comparação entre métodos para avaliação de qualidade fisiológica de sementes de milho. Piracicaba, 1987. 191p. Dissertação (Mestrado) Escola Superior de Agricultura "Luiz de Queiroz", Universidade de São Paulo.

FUTREL, M.C.; KILGORE, M. Poor stands of corn and reduction of root growth caused by Fusarium moniliforme, Beltsville, v.53, p.213-315, 1969.

INTERNATIONAL SEED TESTING ASSOCIATION. Seed health testing. Seed Science and Technology, Zurich, v.4, p.3-49, 1976.

KIEHL, E.J. Manual da edafologia: relaçôes soloplanta. São Paulo: Ceres, 1979. 81p.

LOLLATO, M.A.; SILVA, W.R. da. Efeitos da utilização da mesa gravitacional na qualidade de sementes de feijoeiro. Pesquisa Agropecuaria Brasileira. Brasília, v.19, n.12, p.1483-1496, 1984.

LUCCA FILHO, O.A. Diagnóstico da patologia de sementes de milho no Estado do Rio Grande do Sul. In: SIMPÓSIO BRASILEIRO DE PATOLOGIA DE SEMENTES, 1., 1984, Piracicaba, Situação e perspectivas da patologia de sementes no Brasil. Anais... Piracicaba: CENA/USP/CNEN, Brasilia: ABRATES, 1984. p.102-104.

LUCCA FILHO, O.A. Metodologia dos testes de sanidade de sementes. In: SOAVE, J.; WETZEL, M.V.S. Patologia de sementes, Campinas: Fundação Cargill, 1987a, p.176-198.

LUCCA FILHO, O.A. Teste de sanidade de sementes de milho. In: SOAVE, J.; WETZEL, M.V.S. Patologia de sementes, Campinas: Fundação Cargill, 1987b, p.340-40. 
MELCHERS, L.E.; JOHSTON, C.O. Corn root and ear rot disease investigation in Kansas. Phytopathology, Saint Paul, v.14, p.45, 1924.

MENEZES, M. Aspectos diagnósticos na detecção de Fusarium sp. em sementes. In: SDMPósIO BRASILEIRO DE PATOLOGIA DE SEMENTES, 3 ., 1988. Lavras, Anais... Campinas: Fundação Cargill, 1988, p.140-156.

POPINIGIS, F. Fisiologia da semente. Brasília: AGIPLAN 1977. 289p.

SALAMA, A.M.; MISHRICKY, A.G. Seed transmission of maize wilt fungi with special reference to Fusarium moniliforme. Phytopathologische Zeitachrift, Berlin, v.77, p.356-362, 1973.

SCOTT, G.E.; FUTREL, M.C. Response of maize seedlings to Fusarium moniliforme and a toxic material extracted from this fungus. Plant Disease Reporter. Beltsville, v.54, p.483-486, 1970.

SCOTI, C.A.; KRZYZANOWSKI, F.C. Influência do tamanho da semente sobre a germinação e vigor em milho. Londrina: IAPAR, 1977. 10p. (IAPAR Boletim Técniço, 5).
SILVA, W.R. da. Efeitos do peso e do tamanho das sementes de milho (Zea mays $L$.) sobre a germinação, o vigor e a produção de grãos. Piracicaba, 1978. 83p. Dissertaçāo (Mestrado) - Escola Superior de Agricultura "Luiz de Queiroz", Universidade de São Paulo.

SILVA, W.R. da; MARCOS FILHO, J. Efeitos do peso e do tamanho das sementes de milho sobre a germinaçãoe vigor em laboratório. Revista Brasileira de Sementes, Brasilia, v.1, n.1, p.39-52, 1979.

WARMKE, H.E.; SCHENCK, N.C. Ocurrence of Fusarium moniliforme and Helminthosporium maydis on and in corn seed as related to $T$ cytoplasm. Plant Disease Reporter, Beltswille, v.55, p.486-489, 1971.

ZONTA, E.P.; MACHADO, A.D.; SILVEIRA JUNIOR, $P$. Sistema de análise estatística para microcomputadores - SANEST. Pelotas: UFPel, 1984. (Registro SEI, 06606-0, Categoria, AO).

Trabalho enviado para publicação em 27.08 .92

Trabalho aceito para publicação em 25.11 .92 